\title{
PREDICTION OF THE ELECTIVE SURGERY TIME USING ARTIFICIAL NEURAL NETWORKS
}

\author{
S.Prasanna Devi', S.Sai Sangeetha' ${ }^{2}$ K.Suryaprakasa Rao ${ }^{3}$ \\ Department of Industrial Engineering, \\ College of Engineering, Anna University, India. \\ 1Email: prasanna_siva11@yahoo.com
}

\begin{abstract}
The increasing demand for effective utilization of surgical theatres has put the hospital management system to find ways of improving the capacity utilization of elective surgical suites. The use of artificial neural networks has been widely proved in many healthcare applications. In this light, we undertook a prospective survey of the timing of events of the elective surgeries done in the ophthalmology department and trained the neural network on the target time of the elective surgeries. This research intends to predict the duration of such elective surgeries by means of artificial neural networks which is the first of its kind implemented for forecasting surgery times.
\end{abstract}

Key words:Artificial Neural Networks (ANN), Prediction of Surgery Time, Operation Theatre Utilization.

\section{INTRODUCTION}

The efficient use of operation theatre is very important for several reasons such as reducing the cost while maintaining good quality of service, reporting the efficiency of the surgeon, etc. An accurate prediction of the surgery time for each surgery type is an important parameter for effective planning and utilization of the operation rooms. Such estimation is not an easy task and depends on the pathology of the patient and on the experience of the surgeon. (Wright et al., 1996; Dexter and Macario, 1996). A methodological framework for planning surgery in Operation theatres based on data warehousing and knowledge discovery in databases were proposed by C.Combes et al., 2006 which implements a decision tool to estimate the appropriate time a patient will be in an operation room. A study performed in the endoscopy department by Combes, 2004, indicated that the time taken to perform an endoscopy and the length of time spent by patients in the operating theatre depends on the surgeon, the surgeon's experience in performing the procedure, the type of procedure being performed, and the number of steps involved in the procedure. A considerable amount of research is currently being performed on the subject of planning operating activities, much of this being based on industrial management procedures (Dexter et al.,1999; Shukla et al., 1990; Zhou and Dexter, 1998; Fei et al., 2005;). Combes et al, 2008 have attempted to forecast the surgery time using Knowledge Discovery in Databases. Many publications have been focused on improving the Operation theatre utilization mainly by various scheduling techniques. We aim in predicting the surgery time of an elective opthal surgery using Artificial neural networks taking into account the patient's preconditions, the experience of surgeons, experience of the accompanying medical staffs, type of anesthesia, etc.

\section{METHODS}

An artificial neural network (ANN), often just called a "neural network" (NN), is a mathematical model consisting of an interconnected group of artificial neurons which processes information using a connectionist approach to computation. In most cases an ANN is an adaptive system that changes its structure based on external or internal information that flows through the network. Figure 1 shows the network used for our study. The units combine their inputs to give just one output value. This value is obtained by means of a function knows as a unit activation function. This activation function is made up of two parts. The first part is an aggregation function, which combines all the input values to give a single value. The aggregation function most often used is a weighted sum of inputs. The second part is a transfer function, which transmits the value resulting from the aggregation function to the neuron output. The functions that are most frequently used as transfer functions include sigmoid, linear and hyperbolic tangent functions. Many types of neural networks have been proposed in the literature. The most widespread and best-known is undoubtedly the multilayer perceptron (MLP). This is a feedforward type of neural network, which, as its name suggests, is arranged in layers. MLPs thus typically comprise an input layer (input variables), one or more hidden layers (which are made up of one or more neurons that combine their inputs and generate an output that is passed on to the neurons in the next layer) and an output layer.

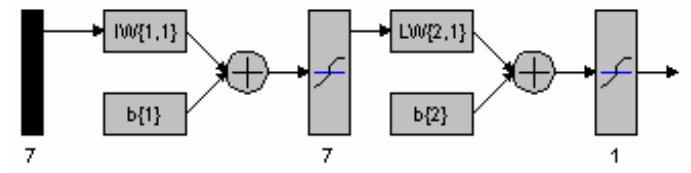

Fig. 1. Artificial Neural Network 
The network architecture shown in Figure 2 has one layer of $S$ neurons connected to $R$ inputs through a matrix of weights $\mathrm{W}$, Where $\mathrm{R}=$ number of elements in input vector and $S=$ number of neurons in layer. Multiple Layers of Neurons" uses layer weight (LW) matrices as well as input weight (IW) matrices. The weight matrix connected to the input vector $p$ is labeled as an input weight matrix $(\operatorname{IW} 1,1)$ having a source 1 and a destination 1 . Elements of layer 1 , such as its bias, net input, and output have a superscript 1 to say that they are associated with the first layer. The configuration of the network used as a basis for the results is as follows: AMLP network including two hidden layers, a linear aggregation function, a tansigmoid activation function, a network learning technique involving standard retropropagation. Other configurations based on modifying the number of hidden layers and the number of neurons in these hidden layers, on changing the aggregation functions (radial, additive, etc.), transfer functions (logistic, exponential) and learning functions, have been tested, but there was no significant improvement in the results.

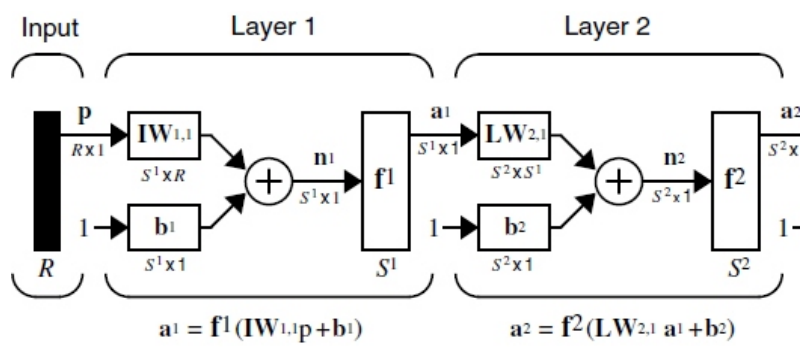

Fig. 2. Network Architecture

\section{DATA COLLECTION}

The data's were collected during the period of March to August 2008 from a multispeciality hospital in India. The following data were obtained from the hospital for each type of elective surgery performed in the Ophthalmology department. The elective surgeries include Corneal Transplants, Cataract, Oculoplastic surgery, etc., This section discusses about the surgery time estimation for Corneal transplants, which is a surgical procedure where a damaged or diseased cornea is replaced by donated corneal tissue.

\section{Table I. Details collected for the study}

1) Start time of surgery,

2) Experience of surgeons performing operation (At most 2 surgeons are involved in a surgery),

3) Experience of accompanying theater staff

4) Type of Anesthesia (Local or General)
5) Experience of Anesthetist,

6) Patient preconditions like existence of Redness of eye, diabetics, hypertension, watery eyes or any other sources of infection.

7) PatientAge

8) Surgery End time

From the above table we can get the actual surgery time which is the difference of the surgery start time and end time. The Graphical user interface, nntool of the MATLAB software has been used for the prediction of surgery time. Table 2 shows the data representation for the Corneal transplant surgery.

Table 2. Data representation for the Corneal Transplant Surgery

\begin{tabular}{|c|c|c|c|c|c|c|c|c|}
\hline \multicolumn{9}{|c|}{ 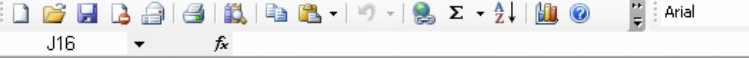 } \\
\hline & A & B & C & D & $E$ & $\mathrm{~F}$ & G & $\mathrm{H}$ \\
\hline 1 & $\begin{array}{l}\text { Patient } \\
\text { Age }\end{array}$ & $\begin{array}{l}\text { Exp of } \\
\text { Surgeon1 }\end{array}$ & $\begin{array}{l}\text { Exp of } \\
\text { Surgeon } \\
2\end{array}$ & $\begin{array}{l}\text { Exp of } \\
\text { accompa } \\
\text { nying } \\
\text { theatre } \\
\text { Staff }\end{array}$ & $\begin{array}{l}\text { Exp of } \\
\text { Anestheti } \\
\text { sts }\end{array}$ & $\begin{array}{l}\text { Type of } \\
\text { Anesthesi } \\
\text { a }\end{array}$ & $\begin{array}{l}\text { Patient } \\
\text { i precondit } \\
\text { ion }\end{array}$ & $\begin{array}{l}\text { Actual } \\
\text { Surgery } \\
\text { Time } \\
\text { (Target } \\
\text { Time in }\end{array}$ \\
\hline 2 & Years & Years & Years & Years & Years & & & hours) \\
\hline 3 & 20 & 5 & 20 & 5 & 7 & 0 & 0 & 1.8 \\
\hline 4 & 45 & 8 & 18 & 8 & 12 & 0 & 3 & 1.3 \\
\hline 5 & 55 & 20 & 5 & 10 & 7 & 0 & 2 & 2 \\
\hline 6 & 37 & 8 & 20 & 8 & 15 & 0 & 3 & 1.9 \\
\hline 7 & 46 & 8 & 18 & 8 & 12 & 1 & 2 & 2.5 \\
\hline 8 & 68 & 20 & 8 & 5 & 15 & 1 & 2 & 2.8 \\
\hline 9 & 29 & 20 & 16 & 10 & 7 & 0 & 1 & 2.3 \\
\hline 10 & 62 & 16 & 10 & 10 & 12 & 1 & 1 & 2.5 \\
\hline 11 & 40 & 5 & 20 & 5 & 15 & 1 & 2 & 2 \\
\hline 12 & 51 & 18 & 8 & 8 & 15 & 0 & 1 & 1.4 \\
\hline 13 & 37 & 8 & 16 & 5 & 12 & 0 & 3 & 1.9 \\
\hline 14 & 56 & 16 & 10 & 5 & 7 & 0 & 1 & 2.3 \\
\hline 15 & 45 & 16 & 8 & 10 & 12 & 0 & 2 & 2 \\
\hline 16 & 37 & 20 & 10 & 8 & 15 & 0 & 3 & 1.8 \\
\hline 17 & 26 & 8 & 10 & 5 & 7 & 1 & 3 & 2.8 \\
\hline 18 & 35 & 16 & 5 & 10 & 12 & 1 & 1 & 2.2 \\
\hline 19 & 43 & 5 & 20 & 8 & 15 & 0 & 2 & 1.7 \\
\hline 20 & 51 & 18 & 6 & 10 & 12 & 0 & 3 & 1.1 \\
\hline 21 & 61 & 10 & 20 & 5 & 12 & 1 & 2 & 2.1 \\
\hline 22 & 47 & 20 & 8 & 8 & 7 & 1 & 2 & 2.7 \\
\hline 23 & 38 & 16 & 10 & 8 & 15 & 1 & 3 & 3 \\
\hline
\end{tabular}

\section{RESULTS \& DISCUSSION}

The input ranges for the 7 input neurons, Patient age, Experience of Surgeon 1, Experience of Surgeon 2, Experience of accompanying theatre staff, Type of Anesthesia (0 - Local, 1 - General), Patient precondition (0 - Redness of eye, 1 - diabetics, 2- hypertension, 3 - other sources of infection) are specified as: [20 70; $325 ; 325 ; 3$ $25 ; 330 ; 01 ; 03]$. The normalized graph of the actual and predicted output is shown in Figure 3 . The variable to be predicted is the surgery time. 


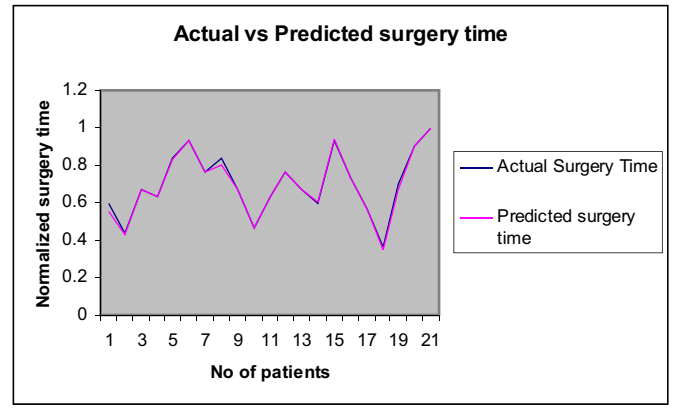

Fig. 3. Graph of Actual vs. predicted surgery time

The results obtained show that the prediction quality is influenced by the choice of variable grouping. From the study conducted in the ophthalmology department, it is evident that the prediction results are influenced by several factors like patient age, experience of surgeons, experience of anesthetist, experience of theatre staff , precondition of the patient etc., Also the Mean Square Error (MSE) is very small which gives a satisfactory prediction accuracy when the predicted values are normalized.

\section{CONCLUSIONS}

In developing countries like India, the hospital resources like the operation theatres are capacitatively constrained. Hence, the hospital management system continuously tries to maximize the effective utilization of such resources. Hence, we intended to study the surgery times for elective surgeries in the ophthalmology department of a multispeciality hospital. This study was used to forecast the surgery time using Artificial Neural Networks. We implemented the prediction for Corneal transplant surgery and found that the prediction quality depends on various factors like patient age, experience of surgeons, experience of anesthetist, experience of theatre staff, precondition of the patient etc., Also the Mean Square Error (MSE) is very small which gives a satisfactory prediction accuracy when the predicted values are normalized.

\section{ACKNOWLEDGEMENT}

We extend our sincere acknowledgements to Dr.Kalpana and Prof. S.P. Thiagarajan of Sri Ramachandra Medical University and Research Centre for providing us with the required data from their Ophthalmology department. We also extend our acknowledgement to Dr.Wang, Associate Professor, Department of System Sciences and Industrial Engineering, Binghamton University for his valuable research suggestions.

\section{REFERENCES}

[1] C. Combes , N. Meskens C. Rivat and J.-P. Vandamme, Using a KDD process to forecast the duration of surgery, International Journal of Production Economics Volume 112, Issue 1, March 2008, Pages 279-293
[2] F. Dexter, A. Macario, R.D. Traub, M. Hopwood and D.A. Lubarsky, An operating room scheduling strategy to maximize the use of operating room block time: computer simulation of patient scheduling and survey of patients' preferences for surgical waiting time, Anesthesia and Analgesia 89 (1999), pp. 7-20.

[3] Health Services Utilization and Research Commission of Saskatchewan, Elective surgery prioritization, URL http://www.sdh.sk.ca/hsurc/ elective.htm (1997).

[4] Mazzei W.J. Operating room start times and turn over times in a university hospital. J clin Anaesth 1994: 405-8.

[5] Principe et al., 2000 J. Principe, N. Euliano and W. Lefebvre, Neural and Adaptive Systems: Fundamentals through Simulations, Wiley, New York (2000).

[6] Ricketts.D, Hartley.J, Patterson.M, Harries.W, Hitchin.D. An orthopaedic theatre timing survey. Ann R Coll Surg Engl 1994; 76(3): 200-4.

[7] Sakharkar.B.M: Operation theatre Suite; In Principles of Hospital Adminsitration and Planning. First edition. Jaypee Bros, New Delhi; 1998: 14; 197-207.

[8] Syed.A.Tabish. Towards development of professional management in Indian hospital. Journal of management in Medicine 1998. 12(2): 109-119.

[9] Vinukondaiah.K, Ananthakrishnan.N, Ravishankar.M. Audit of operation theatre utilization in general surgery. Natl Med J India 2000; 13(3): 11821.

[10] Wright et al., 1996 I.H. Wright, C. Kooperberg, B.A. Bonar and G. Bashein, Statistical modeling to predict elective surgery time, Anesthesiology 85 (1996), pp. 1235-1245

[11] Zhou and Dexter, 1998 J. Zhou and F. Dexter, Method to assist in the scheduling of add-on surgical cases: upper prediction bounds for surgical case durations based on the log normal distribution, Anesthesiology 89 (1998), pp. 1228-1232

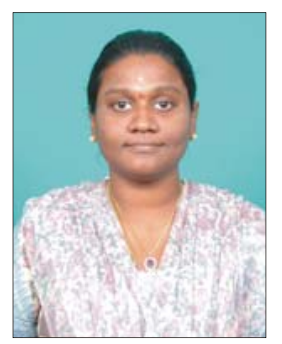

\section{S.Prasanna Devi}

She is currently a Research Scholar in the Industrial Engineering Department, Anna University, Chennai. She has completed her Masters in Systems Engineering \& Operations Research and her undergraduate BE Computer Science from the same University. Her research interests are in the areas of Systems Engineering and Operations Research (SE \& OR). She is working in the area of applications of SE\&OR for the Healthcare management problems. 